\title{
Analysis of Porphyromonas gingivalis fimA genotypes in severe periodontitis patients
}

\section{Richelle Soares RODRIGUES(a) iD Virgínia Régia SILVEIRA ${ }^{(b)}$ iD Rodrigo Otavio REGO(b)}

(a) Universidade Federal do Ceará - UFC, School of Pharmacy, Dentistry and Nursing, Graduate Program in Dentistry, Fortaleza, CE, Brazil.

(b) Universidade Federal do Ceará - UFC, School of Dentistry at Sobral, Department of Dentistry, Sobral, CE, Brazil

Declaration of Interests: The authors certify that they have no commercial or associative interest that represents a conflict of interest in connection with the manuscript.

\section{Corresponding Author:}

Rodrigo Otavio Rego

E-mail: rodrigo.rego@ufc.br

ht1ps://doi.org/10.1590/1807-3107bor-2020.vol34.0090

Submitted: August 31, 2019

Accepted for publication: June 2, 2020

Last revision: June 16, 2020
Abstract: The aim of this study was to i) evaluate the prevalence of $P$. gingivalis and the genotypes fimA I, Ib, II, III, IV, and V in Brazilian patients with periodontitis stage III and IV, grades $B$ and $C$, ii) compare periodontitis grades $B$ and $C$ with regard to the prevalence of $P$. gingivalis and fimA genotypes, and iii) correlate the presence of these pathogens with clinical periodontal variables. Two samples of subgingival biofilm were collected from the interproximal sites with the greatest clinical attachment loss (CAL) of each patient (grade $\mathrm{B}=38$; grade $C=54$ ) and submitted to polymerase chain reaction (PCR) for the identification of $P$. gingivalis and fimA genotypes. The collected periodontal clinical parameters included gingival index, plaque index, probing depth (PD), bleeding on probing (BoP) and CAL. P. gingivalis was present in $61.96 \%$ of the samples, but more prevalent in patients with grade $C$ periodontitis $(p=0.048)$ and higher CAL $(p<0.001), P D$ $(p<0.001)$, and BoP $(p=0.01)$ values, and at sites with high CAL values $(p=0.01)$. The fimA II genotype was more prevalent in patients with greater mean PD $(p=0.04)$ and a higher proportion of bleeding sites $(\mathrm{p}=0.006)$. Thus, in this sample of Brazilian periodontitis patients, the presence of $P$. gingivalis was associated with grade $C$ periodontitis and periodontal destruction, while the fimA II genotype was associated with increased $\mathrm{PD}$ and $\mathrm{BoP}$, supporting the notion that $P$. gingivalis fimA II is an important virulence factor in periodontal tissues.

Keywords: Periodontitis; Porphyromonas gingivalis; Virulence Factors.

\section{Introduction}

Periodontitis is a multifactorial pathology associated with bacterial plaque dysbiosis in the subgingival environment and immunoinflammatory reactions. ${ }^{1,2,3}$ Currently, periodontitis is classified into four stages according to severity and treatment complexity. Stages III and IV are the most severe. Periodontitis can also be classified into grades $\mathrm{A}, \mathrm{B}$, and $\mathrm{C}$ according to the rate of progression and presence of risk factors. ${ }^{3}$

Several pathogens have been associated with periodontal destruction. A periodontal pathogen of Socransky's red complex, ${ }^{4}$ Porphyromonas gingivalis is highly prevalent in the periodontal pockets of periodontitis patients, $5,6,7,7,9,10$ but may also be found in periodontally healthy individuals.,11,12 The great genotypical and phenotypical diversity of $P$. gingivalis results in 
variations in virulence and in the ability to induce periodontal destruction. ${ }^{11}$

The fimbriae are the main structures responsible for the virulent behavior of $P$. gingivalis. They adhere to epithelial cells, fibroblasts, salivary components, and collagen and thus play an important role in the colonization and invasion of periodontal tissues. ${ }^{13}$ The main fimbria is encoded by the fim $A$ gene, allelic variations of which have been correlated with pathogenic potential and specific periodontal conditions.,11

Based on the nucleotide sequence, six genotypes of the fim A gene have been identified ( fim A I, Ib, II, III, IV and V) ${ }^{14,15}$ Several studies have found associations between periodontitis and increased prevalence of fimA II, followed by fimA IV and fimA Ib., ${ }^{911,16,17}$ Likewise, in a study on peri-implantitis, fimA II was the most prevalent genotype, while fim $A \mathrm{Ib}$ was associated with greater peri-implant probing depth. ${ }^{18}$ In another study, the prevalence of $P$. gingivalis and the genotypes fimA II and IV was positively associated with the gingival index in adolescents three months after orthodontic appliance placement. ${ }^{19}$ In contrast, fimA I has been associated with chronic gingivitis not progressing to periodontitis, suggesting this genotype is less virulent. ${ }^{20}$

The knowledge of periodontal pathogens and their association with clinical presentations of periodontitis can help identify individuals at risk for development and progression of periodontal disease. In addition, the distribution of periodontal pathogens and their genotypes is influenced by geographic and ethnic factors. Thus, the present study was designed to meet the following objectives: i) evaluate the prevalence of $P$. gingivalis and the genotypes fim $A \mathrm{I}$, Ib, II, III, IV and V in Brazilian patients diagnosed with periodontitis stage III and IV, grades B and C, ii) compare periodontitis grades $\mathrm{B}$ and $\mathrm{C}$ regarding the prevalence of $P$. gingivalis and fim $A$ genotypes, and iii) correlate the presence of these pathogens with clinical periodontal variables.

\section{Methodology}

This cross-sectional study included 94 patients recruited at the periodontology service of the School of Pharmacy, Dentistry and Nursing of the Federal
University of Ceará (Northeastern Brazil). The study protocol was previously approved by the institutional ethics committee and filed under entry \#20/08. All participants (or guardians) gave their informed written consent.

The patients were initially distributed into two groups (chronic vs. aggressive periodontitis). However, following the new periodontitis classification criteria published in $2018,{ }^{3}$ the patients were reclassified into stages I, II, III, or IV and grades A, B, or C, based on clinical data (clinical attachment loss, probing depth, tooth mobility, furcation involvement, number of remaining teeth, and radiographic bone loss) collected during the periodontal examination. Clinical patterns suggestive of rapid progression and/or early onset of periodontitis and the correlation between plaque deposits and periodontal destruction were used for grading (A, B, or C). In our sample, all patients were stage III or IV and grade B or C. We therefore divided the sample into 'Group B' (stage III/IV, grade B) and 'Group C' (stage III/IV, grade C). The exclusion criteria were: i) periodontal treatment within the previous 6 months, ii) use of antibiotics within the previous 3 months, iii) smoking, and iv) systemic changes capable of interfering with periodontal health.

As stated before, this study was originally designed to evaluate differences between the prevalence of $P$. gingivalis fim $A$ genotypes in patients with aggressive periodontitis and chronic periodontitis, characteristics of the previous classification of periodontal diseases. Then, using data from a pilot study, ${ }^{5}$ and considering a ratio of 0.16 between fimA II negative to positive patients, a sample size of at least 28 patients with aggressive periodontitis would be needed to provide $80 \%$ power with $\alpha=0.05$ (Epi-Info ${ }^{\mathrm{TM}}, \mathrm{CDC}$, Atlanta, GA, USA).

\section{Clinical measurements}

All completely erupted permanent teeth (except third molars) were evaluated using a periodontal probe (PCP-UNC 15, Trinity, São Paulo, Brazil). The clinical parameters included: plaque index $(\mathrm{PI})^{21}$ gingival index $(\mathrm{GI})^{21}$, probing depth (PD), clinical attachment loss (CAL), bleeding on probing (BoP), tooth mobility, and furcation involvement. PD and CAL were taken at six sites per tooth (mesiobuccal, buccal, distobuccal, mesiolingual, lingual, and distolingual). 
A single examiner, a calibrated periodontist, evaluated all clinical parameters. For reproducibility analysis, duplicate measurements were performed on two occasions, one prior to initiating the study and a second during the study. Twelve individuals diagnosed with periodontitis stage III or IV were evaluated twice, with a three-day interval. Data were analyzed, and the intraclass correlation coefficient (ICC) was 0.88 and 0.83 for PD and CAL measurements, respectively.

\section{Microbiological analysis}

Supragingival plaque was removed with curettes and sterile cotton pellets, and the area was isolated with sterile cotton rolls. Subgingival plaque samples were then collected from two different sites per patient using two sterile paper points (Dentsply Maillefer 35, Dentsply, Rio de Janeiro, Brazil) per site, inserted for 20 seconds. ${ }^{22}$ The proximal site with the greatest PD and CAL of molars or incisors was selected for each patient. Each sample was placed in a microtube containing $1 \mathrm{~mL}$ Ringer's solution $(8.6 \mathrm{~g} \mathrm{NaCl}, 0.3 \mathrm{~g}$ $\mathrm{KCl}$, and $0.33 \mathrm{~g} \mathrm{CaCl} 2 \mathrm{HO}$ in $1000 \mathrm{~mL} \mathrm{HO}$ ) and stored at $-80^{\circ} \mathrm{C}$ until the time of use.

Each sample was processed separately. The samples were thawed on ice, and bacterial cells were dispersed by vortexing at the maximum setting for $1 \mathrm{~min}$, then centrifuged at $12,000 \mathrm{~g}$ for $10 \mathrm{~min}$. Genomic DNA was extracted from the pellet following the manufacturer's instructions (InstaGene Matrix, Bio-Rad Laboratories, Hercules, USA). A $20-\mu \mathrm{L}$ aliquot of the resulting supernatant was added to $30 \mu \mathrm{L}$ reaction mixture containing $25 \mu \mathrm{M}$ PCR buffer (Promega Corporation, Madison, USA), $25 \mu \mathrm{M} \mathrm{MgCl}_{2}$ (Promega Corporation), $0.2 \mu \mathrm{M}$ dNTP mix (Promega Corporation), 1.25 U Taq polymerase (Promega Corporation), and $100 \mathrm{ng}$ of each primer (Invitrogen, São Paulo, Brazil), resulting in a final volume of $50 \mu \mathrm{L} .{ }^{22,23}$ Negative and positive controls were included in each reaction (Table 1).

First, PCR was performed with universal primers ${ }^{24}$ for $16 \mathrm{~S}$ ribosomal DNA (16S rDNA) to confirm the presence of bacterial DNA. Subsequently, samples were evaluated by PCR with specific primers for the presence of $P$. gingivalis ${ }^{25}$ and fim $A$ genotypes $\mathrm{I}^{14} \mathrm{Ib}^{15} \mathrm{II}^{14}{ }^{14} \mathrm{II},{ }^{14} \mathrm{IV}^{14}$ and V. ${ }^{26}$ Amplification was performed with a biocycler (Biosystems, Curitiba,
Table 1. Strains used as positive control.

\begin{tabular}{lc}
\hline Clonal type & Strain \\
\hline fimA I & P. gingivalis ATCC 33277 \\
fimA Ib & P. gingivalis HG 1691 \\
fimA II & P. gingivalis HW24D-1 \\
fimA III & P. gingivalis 6/26 \\
fimA IV & P. gingivalis HG564 \\
fimA V & P. gingivalis HNA99 \\
\hline
\end{tabular}

Brazil), followed by analysis of the products by electrophoresis on agarose gel. The gels were stained with SYBR ${ }^{\circledR}$ Safe (Invitrogen, São Paulo, SP, Brazil) and photographed (Canon Powershot A640, Canon, USA) under ultraviolet light (LTA/LTB GE, Loccus Biotecnologia, São Paulo, Brazil). PCR was repeated three times for each sample and for P.gingivalis and its genotypes.

\section{Statistical analysis}

The normality of the data distribution was verified with the Shapiro-Wilk test. Comparisons between Group B and Group C with regard to age, clinical data, and the presence of $P$. gingivalis and its genotypes were made with the unpaired $t$ test and the Mann-Whitney U test. Pearson's chi-squared test was used to compare the groups with regard to the prevalence of $P$. gingivalis and fim $A$ genotypes. The level of statistical significance was set at $5 \%(p<0.05)$. All analyses were performed with the software SPSS 25.0 (IBM Corp., Armonk, USA).

\section{Results}

Fifty patients were classified as stage III and 44 were classified as stage IV. As for grading, 40 subjects were graded ' $B$ ' and 54 subjects were graded ' $C$ '. Two grade $B$ patients yielded samples that could not be amplified, leaving 38 patients in Group B vs. 54 patients in Group C. Table 2 shows the demographic and clinical data of all 92 patients. The patients in Group C were significantly younger, had more teeth, and greater PD and BoP values than the patients in Group B.

P. gingivalis was present in 57 patients (61.96\%), but was more prevalent in Group C than in Group B $(p=0.048)$. The groups did not differ significantly with regard to the prevalence of fimA genotypes (Table 3). 
Table 2. Characteristics of the study participants.

\begin{tabular}{|c|c|c|c|}
\hline Variables & Group B & Group C & n nolus \\
\hline $\mathrm{N}$ & 38 & 54 & $p$-varue \\
\hline Age (years; mean $\pm S D$ ) & $41.82 \pm 8.49$ & $27.94 \pm 6.15$ & $<0.001^{*}$ \\
\hline Male/female & $16 / 22$ & $20 / 34$ & 0.62 \\
\hline White/non-white & $11 / 27$ & $11 / 43$ & 0.85 \\
\hline Number of teeth (mean \pm SD) & $23.32 \pm 3.17$ & $25.94 \pm 1.89$ & $<0.001^{*}$ \\
\hline $\mathrm{PD}(\mathrm{mm} ;$ mean $\pm \mathrm{SD})$ & $2.90 \pm 0.64$ & $3.42 \pm 0.68$ & $<0.001^{*}$ \\
\hline $\mathrm{CAL}(\mathrm{mm} ;$ mean $\pm \mathrm{SD})$ & $3.54 \pm 1.04$ & $3.81 \pm 0.89$ & 0.17 \\
\hline BoP (\% sites; mean $\pm \mathrm{SD}$ ) & $33.46 \pm 17.07$ & $45.00 \pm 16.67$ & $0.02^{*}$ \\
\hline PD (sampled site 1) $(\mathrm{mm}$; mean $\pm \mathrm{SD})$ & $7.71 \pm 2.09$ & $8.42 \pm 2.18$ & 0.12 \\
\hline $\mathrm{CAL}$ (sampled site 1$)(\mathrm{mm}$; mean $\pm \mathrm{SD}$ ) & $8.79 \pm 2.41$ & $9.31 \pm 2.48$ & 0.31 \\
\hline $\mathrm{PD}$ (sampled site 2$)(\mathrm{mm}$; mean $\pm \mathrm{SD})$ & $6.60 \pm 1.53$ & $7.28 \pm 2.06$ & 0.09 \\
\hline CAL (sampled site 2$)(\mathrm{mm}$; mean $\pm \mathrm{SD}$ ) & $7.31 \pm 1.72$ & $8.02 \pm 2.54$ & 0.14 \\
\hline
\end{tabular}

Table 3. Prevalence of $P$. gingivalis and $\operatorname{fim} A$ genotypes according to group.

\begin{tabular}{lccc}
\hline \multirow{2}{*}{ Pg } & Group B & Group C & p-value \\
\cline { 2 - 4 } & $19(50.0 \%)$ & $38(70.4 \%)$ & $0.048^{*}$ \\
\hline fimA I & $6(31.6 \%)$ & $6(15.8 \%)$ & 0.17 \\
fimA Ib & $6(31.6 \%)$ & $7(18.4 \%)$ & 0.26 \\
fimA II & $16(84.2 \%)$ & $35(92.1 \%)$ & 0.36 \\
fimA III & $1(5.3 \%)$ & $1(2.6 \%)$ & 0.61 \\
fimA IV & $1(5.3 \%)$ & $0(0.0 \%)$ & 0.15 \\
fimA V & $0(0.0 \%)$ & $1(2.6 \%)$ & 0.48 \\
\hline * & & &
\end{tabular}

Patients positive for $P$. gingivalis presented significantly higher mean PD, CAL, interproximal CAL, and BoP, and greater PD and CAL at selected sites, than did $P$. gingivalis-negative patients. The mean PD and BoP values were also higher among fimA II-positive than fimA II-negative patients. No significant associations were found between clinical variables and the five other fimA genotypes. Moreover, the proportion of selected sites with means for PD $\geq 7 \mathrm{~mm}, \mathrm{PD} \geq 8 \mathrm{~mm}$, and CAL $\geq 8 \mathrm{~mm}$ was significantly greater for $P$. gingivalis-positive than $P$. gingivalis-negative patients (Table 4).

\section{Discussion}

Our study was originally designed to evaluate the possible correlations between aggressive vs. chronic periodontitis and $P$. gingivalis fim $A$ genotypes. However, in 2018, the World Workshop on the Classification of
Periodontal and Peri-Implant Diseases and Conditions concluded there is insufficient evidence to support the concept of aggressive and chronic periodontitis as two pathophysiologically distinct diseases. ${ }^{3,27} \mathrm{~A}$ new classification system was proposed, according to which periodontitis is no longer divided into chronic and aggressive but classified into stages and grades based on clinical parameters and other elements influencing clinical management, prognosis, and, potentially, both oral and systemic health..$^{3,27}$

Thus, all patients were reclassified as stage III or IV primarily based on the finding of interproximal $C A L \geq 5 \mathrm{~mm}$ and $P D \geq 6 \mathrm{~mm}$. The classification also took into account dental mobility $(\geq 2)$, furcation involvement (class II or III), the number of remaining teeth, and radiographic bone loss. 3,27

Due to the cross-sectional design of the study, no direct data on disease progression was collected, but indirect evidence of progression was used to grade the patients as A, B, or C. Smoking habits and systemic disease were exclusion criteria and so were not included in the analysis. None of our patients presented deposits of heavy biofilm with low levels of destruction (Grade A). When destruction exceeded expectations for the level of biofilm deposits and a specific clinical pattern was observed (e.g., incisive/molar pattern or early-onset disease), the patient was graded ' $C$ '. When destruction matched expectations for the level of biofilm deposits, with no specific clinical pattern suggestive of grade $\mathrm{A}$ or $\mathrm{C}$, the patient was graded ' $\mathrm{B}{ }^{\prime}$. 


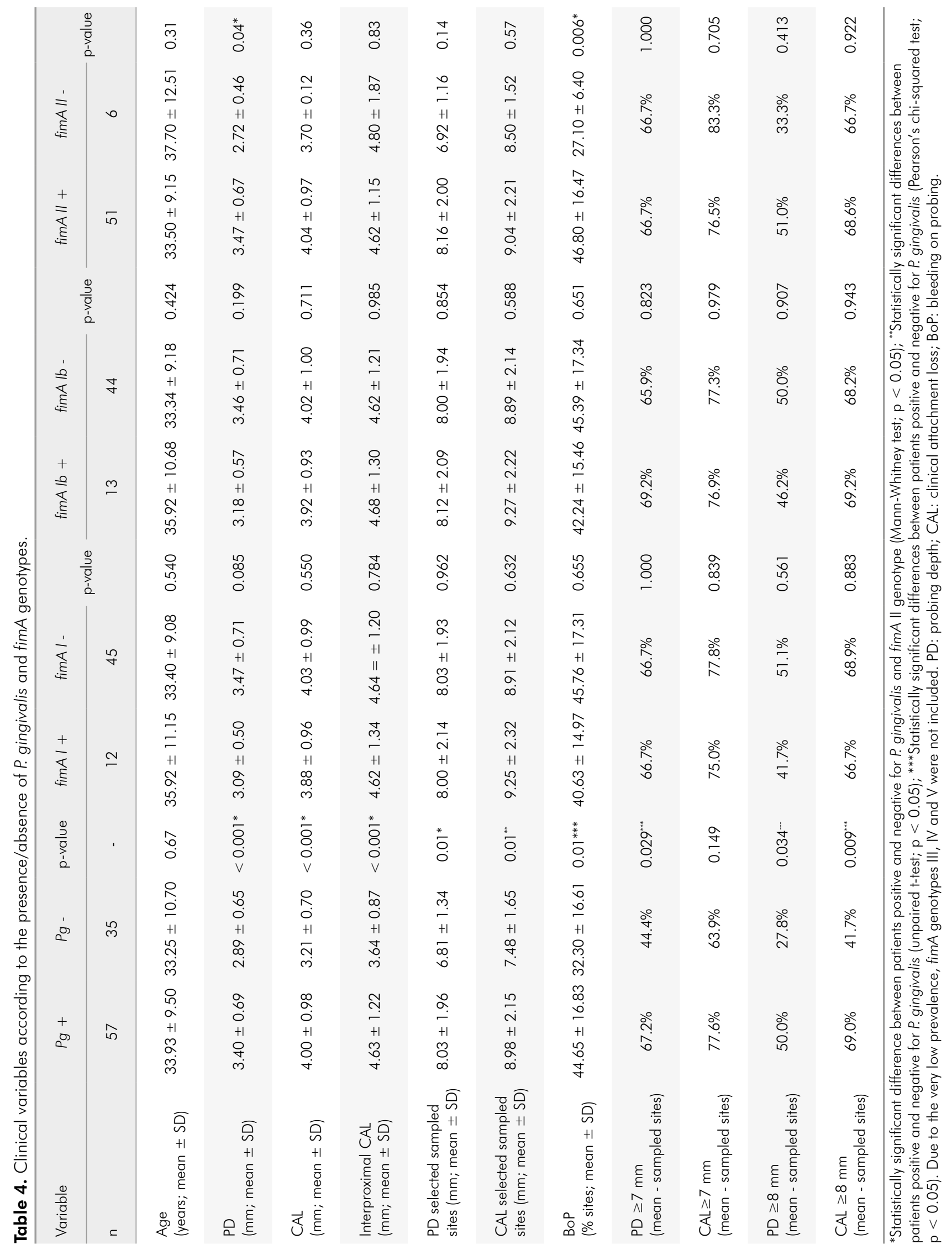


The patients were organized in groups according to grade, not stage. While the stage depends on the severity and extent of the disease at the time of presentation, the grade provides information on biological features of the disease such as specific clinical patterns suggestive of periods of rapid progression and/or early-onset of the disease and the relationship between plaque deposits and periodontal destruction, ${ }^{3,27}$ as previously required for the diagnosis of aggressive periodontitis..$^{28,29}$

The mean age was higher, and the mean number of teeth was smaller in Group B than in Group C. Despite the lower mean age in Group $C$, the mean $\mathrm{BoP}$ value was higher, suggesting greater disease severity. This was expected since Group C consisted of patients with destruction patterns suggestive of periods of rapid progression and early-onset of disease, especially those previously classified as having aggressive periodontitis.

In our sample, the high prevalence of $P$. gingivalis was associated with periodontitis stage III and IV, especially grade $C$. This finding matches the results of a recent metanalysis of 42 case-control studies showing a $78.7 \%$ prevalence of $P$. gingivalis in periodontal disease, ${ }^{30}$ although the reported prevalence rates vary widely in the literature. ${ }^{711,17,31,32}$ The prevalence of $P$. gingivalis was higher in Group C than in Group B. Since the classification of periodontitis into stages and grades was introduced very recently, no data was available for comparison. Nevertheless, the presence of $P$. gingivalis has been positively correlated with greater probing depth and periodontitis severity. ${ }^{17,32,33}$ Likewise, in our study, Group C was associated with greater mean probing depth and the proportion of sampled sites with $\mathrm{PD}$ and CAL $\geq 8 \mathrm{~mm}$ was greater in $P$. gingivalis-positive than $P$. gingivalis-negative patients.

Among P. gingivalis-positive samples, the fimA II genotype was the most prevalent regardless of group $(B=84.2 \% ; C=92.1 \%)$, matching a previous Brazilian study on a sample of patients with aggressive periodontitis. ${ }^{5}$ However, the prevalence of the fimA II genotype was higher in our study than in many other studies involving patients with chronic and aggressive periodontitis in a variety of populations from Japan, 9,26 Germany, ${ }_{17}^{17}$ and even Brazil. ${ }^{11}$ When only patients with aggressive periodontitis (equivalent to grade $\mathrm{C}$ ) were considered, the prevalence of the fimA II genotype was still substantially higher than that reported for Japanese ${ }^{7}$ and Chinese cohorts. ${ }^{31}$ These discrepancies may be explained by differences in the severity of periodontal disease and by ethnic and geographic variability. In any case, the fimA II genotype very likely played an important role in the development and progression of periodontitis in our sample of Brazilian patients.

Despite the higher prevalence of fimA II and the lower prevalence of fim $A$ I in group $C$, the difference between the groups was not statistically significant. This trend has been observed in several other studies, invariably associating the fimA II genotype with greater virulence and periodontal destruction, $, 5,9,11,17,31$ whereas the fim A I genotype has been associated with lower virulence and healthy patients. ${ }^{9,31}$ Since Group C consisted mainly of patients previously diagnosed with aggressive periodontitis, our findings support the results of Japanese and Chinese studies on aggressive periodontitis. ${ }^{7,31}$ The absence of statistical significance may be due to the narrow range of disease severity in the sample: both groups consisted of patients with advanced periodontal disease (stages III and IV).

In addition to the high prevalence of $P$. gingivalis and fimA II, the periodontal clinical variables (such as mean PD and BoP) were also more severe in $P$. gingivalis-positive patients.

P. gingivalis of the fimA II genotype is related to the progression of periodontal disease, ${ }^{35}$ adhering to and invading human epithelial cells and inducing inflammation more efficiently than any other genotype. ${ }^{13,35,36,37}$ The presence of fimA II is therefore believed to be an important $P$. gingivalis virulence factor in periodontal tissues. ${ }^{34,35,36,37}$ The detection of this genotype can help identify individuals at risk, make adequate treatment plans, and establish a more accurate prognosis. In addition, studies on the fim $\mathrm{A}$ II genotype are crucial to the development of new treatment strategies such as passive immunization with monoclonal antibodies from $P$. gingivalis fimA II strains, as demonstrated by Hijiya et al..$^{38}$ in a rat model.

\section{Conclusions}

In our sample of Brazilian patients with periodontitis stages III and IV, the prevalence of 
P. gingivalis was higher in patients graded ' $\mathrm{C}$ ' than ' $\mathrm{B}$ ' and in patients with severe periodontal destruction as expressed by greater clinical attachment loss, probing depth, and bleeding on probing. Moreover, the prevalence of the fimA II genotype was higher in patients with greater mean probing depth and a greater proportion of bleeding sites.

\section{Acknowledgments}

This study was supported by grants from $\mathrm{CNPq}$ (478161/2007-7) and CAPES (PROCAD NF 2313/2008). We would also like to thank the Oral Microbiology Group at the Academic Centre for Dentistry Amsterdam (ACTA) for providing Porphyromonas gingivalis strains.

\section{References}

1. Kantarci A, Hasturk H, Van Dyke TE. Host-mediated resolution of inflammation in periodontal diseases. Periodontol 2000. 2006;40(1):144-63. https://doi.org/10.1111/j.1600-0757.2005.00145.x

2. Kinane DF, Shiba H, Hart TC. The genetic basis of periodontitis. Periodontol 2000. 2005;39(1):91-117. https://doi.org/10.1111/j.1600-0757.2005.00118.x

3. Papapanou PN, Sanz M, Buduneli N, Dietrich T, Feres M, Fine DH, et al. Periodontitis: Consensus report of workgroup 2 of the 2017 World Workshop on the Classification of Periodontal and Peri-Implant Diseases and Conditions. J Clin Periodontol. 2018 Jun;45 Suppl 20:S162-70. https://doi.org/10.1111/icpe.12946

4. Socransky SS, Haffajee AD, Cugini MA, Smith C, Kent RL Jr. Microbial complexes in subgingival plaque. J Clin Periodontol. 1998 Feb;25(2):134-44. https://doi.org/10.1111/j.1600-051X.1998.tb02419.x

5. Rodrigues RS, Tahim CM, Silveira VR, Nogueira NA, Rego RO. Prevalence of Porphyromonas gingivalis fimA II genotype in generalized aggressive periodontitis patients. Braz J Oral Sci. 2016 Jul./Set.;15(3):176-80. https://doi.org/10.20396/bjos.v15i3.8649601

6. Casarin RC, Ribeiro EP, Mariano FS, Nociti FH Jr, Casati MZ, Gonçalves RB. Levels of Aggregatibacter actinomycetemcomitans, Porphyromonas gingivalis, inflammatory cytokines and species-specific immunoglobulin $G$ in generalized aggressive and chronic periodontitis. J Periodontal Res. 2010 Oct;45(5):635-42. https://doi.org/10.1111/j.1600-0765.2010.01278.x

7. Miura M, Hamachi T, Fujise $O$, Maeda K. The prevalence and pathogenic differences of Porphyromonas gingivalis fimA genotypes in patients with aggressive periodontitis. J Periodontal Res. 2005 Apr;40(2):147-52. https://doi.org/10.1111/j.1600-0765.2005.00779.x

8. Silva-Boghossian CM, Souto RM, Luiz RR, Colombo AP. Association of red complex, A. actinomycetemcomitans and non-oral bacteria with periodontal diseases. Arch Oral Biol. 2011 Sep;56(9):899-906. https://doi.org/10.1016/i.archoralbio.2011.02.009

9. Amano A, Kuboniwa M, Nakagawa I, Akiyama S, Morisaki I, Hamada S. Prevalence of specific genotypes of Porphyromonas gingivalis fimA and periodontal health status. J Dent Res. 2000 Sep;79(9):1664-8. https://doi.org/10.1177/00220345000790090501

10. Fabrizi S, León R, Blanc V, Herrera D, Sanz M. Variability of the fimA gene in Porphyromonas gingivalis isolated from periodontitis and non-periodontitis patients. Med Oral Patol Oral Cir Bucal. 2013 Jan;18(1):e100-5. https://doi.org/10.4317/medoral.18042

11. Missailidis CG, Umeda JE, Ota-Tsuzuki C, Anzai D, Mayer MP. Distribution of fimA genotypes of Porphyromonas gingivalis in subjects with various periodontal conditions. Oral Microbiol Immunol. 2004 Aug;19(4):224-9. https://doi.org/10.1111/j.1399-302X.2004.00140.x

12. Zhao L, Wu YF, Meng S, Yang H, OuYang YL, Zhou XD. Prevalence of fimA genotypes of Porphyromonas gingivalis and periodontal health status in Chinese adults. J Periodontal Res. 2007 Dec;42(6):511-7. https://doi.org/10.1111/i.1600-0765.2007.00975.x

13. Hamada S, Amano A, Kimura S, Nakagawa I, Kawabata S, Morisaki I. The importance of fimbriae in the virulence and ecology of some oral bacteria. Oral Microbiol Immunol. 1998 Jun;13(3):129-38. https://doi.org/10.1111/j.1399-302X.1998.tb00724.x

14. Amano A, Nakagawa I, Kataoka K, Morisaki I, Hamada S. Distribution of Porphyromonas gingivalis strains with fimA genotypes in periodontitis patients. J Clin Microbiol. 1999 May;37(5):1426-30. https://doi.org/10.1128/JCM.37.5.1426-1430.1999

15. Nakagawa I, Amano A, Ohara-Nemoto Y, Endoh N, Morisaki I, Kimura S, et al. Identification of a new variant of fimA gene of Porphyromonas gingivalis and its distribution in adults and disabled populations with periodontitis. J Periodontal Res. 2002 Dec;37(6):425-32. https://doi.org/10.1034/i.1600-0765.2002.01637.x

16. Tamura K, Nakano K, Nomura R, Miyake S, Nakagawa I, Amano A, et al. Distribution of Porphyromonas gingivalis fimA genotypes in Japanese children and adolescents. J Periodontol. 2005 May;76(5):674-9. https://doi.org/10.1902/jop.2005.76.5.674

17. Beikler T, Peters U, Prajaneh S, Prior K, Ehmke B, Flemmig TF. Prevalence of Porphyromonas gingivalis fimA genotypes in Caucasians. Eur J Oral Sci. 2003 Oct;111(5):390-4. https://doi.org/10.1034/i.1600-0722.2003.00065.x

18. Kim SG, Hong JY, Shin S. II, Moon JH, Lee JY, Herr Y. Prevalence of Porphyromonas gingivalis fimA genotypes in periimplant sulcus of Koreans using new primer. J Periodontal Implant Sci. 2016 Feb;46(1):35-45. https://doi.org/10.5051/ipis.2016.46.1.35 
- Analysis of Porphyromonas gingivalis fimA genotypes in severe periodontitis patients

19. Pan S, Liu Y, Si Y, Zhang Q, Wang L, Liu J, et al. Prevalence of fimA genotypes of Porphyromonas gingivalis in adolescent orthodontic patients. PLoS One. 2017 Nov;12(11):e0188420. https://doi.org/10.1371/journal.pone.0188420

20. Krishnan M, Krishnan P, Chandrasekaran SC. Detection of Porphyromonas gingivalis fimA type I genotype in gingivitis by real-time PCR: a pilot study. J Clin Diagn Res. 2016 Jun;10(6):ZC32-5. https://doi.org/10.7860/JCDR/2016/17938.7979

21. Ainamo J, Bay I. Problems and proposals for recording gingivitis and plaque. Int Dent J. 1975 Dec;25(4):229-35.

22. Silveira VR, Nogueira MV, Nogueira NA, Lima V, Furlaneto FA, Rêgo RO. Leukotoxicity of Aggregatibacter actinomycetemcomitans in generalized aggressive periodontitis in Brazilians and their family members. J Appl Oral Sci. 2013 Sep-Oct;21(5):430-6. https://doi.org/10.1590/1679-775720130252

23. Silveira VR, Pigossi SC, Scarel-Caminaga RM, Cirelli JA, Rêgo R, Nogueira NA. Analysis of polymorphisms in Interleukin 10, NOS2A, and ESR2 genes in chronic and aggressive periodontitis. Braz Oral Res. 2016 Oct;30(1):e105. https://doi.org/10.1590/1807-3107BOR-2016.vol30.0105

24. Wilson KH, Blitchington RB, Greene RC. Amplification of bacterial 16S ribosomal DNA with polymerase chain reaction. J Clin Microbiol. 1990 Sep;28(9):1942-6. https://doi.org/10.1128/JCM.28.9.1942-1946.1990

25. Tran SD, Rudney JD. Multiplex PCR using conserved and species-specific 16S rRNA gene primers for simultaneous detection of Actinobacillus actinomycetemcomitans and Porphyromonas gingivalis. J Clin Microbiol. 1996 Nov;34(11):2674-8. https://doi.org/10.1128/JCM.34.11.2674-2678.1996

26. Nakagawa I, Amano A, Kimura RK, Nakamura T, Kawabata S, Hamada S. Distribution and molecular characterization of Porphyromonas gingivalis carrying a new type of fimA gene. J Clin Microbiol. 2000 May;38(5):1909-14. https://doi.org/10.1128/JCM.38.5.1909-1914.2000

27. Tonetti MS, Greenwell H, Kornman KS. Staging and grading of periodontitis: framework and proposal of a new classification and case definition. J Clin Periodontol. 2018 Jun;45 Suppl 20:S149-61. https://doi.org/10.1111/icpe.12945

28. American Academy of Periodontology. Parameter on aggressive periodontitis. J Periodontol. 2000;71(5S):867-9. https://doi.org/10.1902/jop.2000.71.5-S.867

29. Brito LF, Taboza ZA, Silveira VR, Furlaneto FA, Rosing CK, Rego RO. Aggressive periodontitis presents a higher degree of bilateral symmetry in comparison with chronic periodontitis. J Oral Sci. 2018;60(1):97-104. https://doi.org/10.2334/josnusd.16-0669

30. Rafiei M, Kiani F, Sayehmiri K, Sayehmiri F, Tavirani M, Dousti M, et al. Prevalence of anaerobic bacteria (P.gingivalis) as major microbial agent in the incidence periodontal diseases by meta-analysis. J Dent (Shiraz). 2018 Sep;19(3):232-42.

31. Feng $X$, Zhang L, Xu L, Meng H, Lu R, Chen Z, et al. Detection of eight periodontal microorganisms and distribution of Porphyromonas gingivalis fimA genotypes in Chinese patients with aggressive periodontitis. J Periodontol. 2014 Jan;85(1):150-9. https://doi.org/10.1902/jop.2013.120677

32. Abreu MG, Kawamoto D, Mayer MP, Pascoal VD, Caiaffa KS, Zuza EP, et al. Frequency of Porphyromonas gingivalis fimA in smokers and nonsmokers after periodontal therapy. J Appl Oral Sci. 2019;27:e20180205. https://doi.org/10.1590/1678-7757-2018-0205

33. Chigasaki O, Takeuchi Y, Aoki A, Sasaki Y, Mizutani K, Aoyama N, et al. A cross-sectional study on the periodontal status and prevalence of red complex periodontal pathogens in a Japanese population. J Oral Sci. 2018;60(2):293-303. https://doi.org/10.2334/josnusd.17-0223

34. Puig-Silla M, Montiel-Company JM, Dasí-Fernández F, Almerich-Silla JM. Prevalence of periodontal pathogens as predictor of the evolution of periodontal status. Odontology. 2017 Oct;105(4):467-76. https://doi.org/10.1007/s10266-016-0286-x

35. Nakano K, Kuboniwa M, Nakagawa I, Yamamura T, Nomura R, Okahashi N, et al. Comparison of inflammatory changes caused by Porphyromonas gingivalis with distinct fimA genotypes in a mouse abscess model. Oral Microbiol Immunol. 2004 Jun;19(3):205-9. https://doi.org/10.1111/i.0902-0055.2004.00133.x

36. Gao L, Xu Y, Meng S, Wu Y, Huang H, Su R, et al. Identification of the putative specific pathogenic genes of Porphyromonas gingivalis with type II fimbriae. DNA Cell Biol. 2012 Jun;31(6):1027-37. https://doi.org/10.1089/dna.2011.1487

37. Kato T, Kawai S, Nakano K, Inaba H, Kuboniwa M, Nakagawa l, et al. Virulence of Porphyromonas gingivalis is altered by substitution of fimbria gene with different genotype. Cell Microbiol. 2007 Mar;9(3):753-65. https://doi.org/10.1111/j.1462-5822.2006.00825.x

38. Hiijiya T, Shibata Y, Hayakawa M, Abiko Y. A monoclonal antibody against fimA type II Porphyromonas gingivalis inhibits IL-8 production in human gingival fibroblasts. Hybridoma (Larchmt). 2010 Jun;29(3):201-4. https://doi.org/10.1089/hyb.2009.0109 